\section{Advances in brain metastases presented at the American Society of Clinical Oncology 2016 Annual Meeting: Part II}

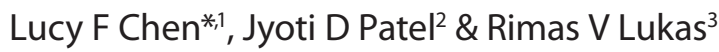

\begin{abstract}
American Society of Clinical Oncology Annual Meeting, Chicago, IL, USA, 3-7 June 2016
The American Society of Clinical Oncology Annual Meeting took place in Chicago, IL, USA, from 3 to 7 June 2016. Over 30,000 oncologists, researchers, related professionals and advocates participated in the conference, which covered all aspects of oncology. An overview of the key studies in brain metastases presented at the 2016 American Society of Clinical Oncology Annual Meeting is highlighted here. Key data presented on radiotherapy, and systemic therapy for brain metastases are reviewed.
\end{abstract}

First draft submitted: 12 August 2016; Accepted for publication: 23 August 2016; Published online: 8 September 2016

\section{Radiotherapy}

Whole-brain radiotherapy (WBRT) has been the cornerstone of brain metastases (BM) treatment for decades. The Japanese study JCOG0504 sought to better understand the role of stereotactic radiosurgery (SRS) compared with WBRT after surgical resection for 1-4 BM. This study evaluated 271 patients (Eastern Oncology Cooperative Group [ECOG] performance status 0-3) with lung (non-small-cell lung cancer [NSCLC]), breast, colon, kidney, ovary, uterine, stomach and esophagus primary sites with 1-4 BM. Surgery was performed for tumors with a maximum size greater than $3 \mathrm{~cm}$. Within 21 days after surgery, 137 patients were randomized to WBRT (37.5 Gy in 15 fractions) and 134 patients to SRS (marginal dose $24 \mathrm{~Gy}[<2 \mathrm{~cm}], 18 \mathrm{~Gy}[2.1-3 \mathrm{~cm}])$. The baseline characteristics between the WBRT and SRS groups were well balanced with the primary endpoint being overall survival (OS). The median OS in both groups was 15.6 months with a hazard ratio of $1.05(90.0 \% \mathrm{CI}: 0.83-1.33$; one-sided $\mathrm{p}=0.027)$. The intracranial progressionfree survival was significantly longer in the WBRT group (10.4 months) compared with the SRS group ( 4.0 months; $\mathrm{p}<0.0001)$. The proportion of patients with grade $2-4$ cognitive dysfunction at more than 3 months from trial enrollment was significantly higher in the WBRT group (16.4 vs 7.7\%) [1]. This study demonstrated noninferiority of SRS to WBRT consistent with prior data.

A multi-institutional analysis evaluated the role of surgery added to SRS in patients with large BM ( $\geq 4$ cc or 2-cm diameter). Between 2005 and 2013, 250 patients with 260 treated large BM were included. Treatment was with single fraction SRS alone or surgery with either pre- or post-operative SRS. Preoperative SRS dose was 20\% lower than the postoperative SRS dose. 194 patients were treated with surgery and SRS boost while 66 were treated with SRS alone. The Kaplan-Meier method

\section{KEYWORDS}

- brain metastases $\bullet$ cancer metastases $\bullet$ chemotherapy

- immunotherapy

- radiotherapy $\bullet$ targeted therapies 
was used to analyze OS. The median imaging follow-up was 29 months for alive patients. The two groups were well balanced with a few exceptions. The SRS alone group had lower lesion volume (gross tumor volume, median 5.9 vs $9.3 \mathrm{cc}$; $\mathrm{p}<0.001)$, higher median number of BM (1.5 vs $1 ; \mathrm{p}=0.006)$ and higher median SRS dose (18 vs $15 \mathrm{~Gy} ; \mathrm{p}=0.001)$. OS was significantly higher with SRS boost (2-year OS: 35.5 vs $19.5 \%$; $\mathrm{p}=0.013)$, and local recurrence (LR) was significantly lower with SRS boost (1-year LR: 36.7 vs $18.8 \% ; \mathrm{p}=0.004)$. Both remained significant in multivariate analysis. There was significantly increased radiation necrosis (RN) in the postoperative SRS compared with the pre-operative SRS and SRS alone groups in multivariate analysis (hazard ratio: 2.9; 95\% CI: 1.04-8.5; $\mathrm{p}=0.05$, 1-year RN: 22, 4.8, 12.3\%; p < 0.001, respectively) [2]. While this demonstrates that surgical resection with SRS boost may improve OS and decrease LR for patients with limited number of large $\mathrm{BM}$, the increased rate of $\mathrm{RN}$ will need to be factored and data regarding whether this RN was clinically significant will be important.

\section{Systemic therapies}

The treatment of BM with systemic therapies has been limited by the blood-brain barrier and by tumor-related factors (including size, number, chemosensitivity and tumor heterogeneity). A novel peptide drug conjugate, ANG1005, showed promise with data from the ANG1005-CLN-04 trial. ANG1005 is a taxane derivative designed to cross the blood-brain barrier via the LRP-1 transport system. In this multicenter, open-label study, 72 patients with recurrent BM from breast cancer were enrolled (those with leptomeningeal metastases [LM] were also included). Intracranial objective response rate was the primary end point. ANG1005 was administered at $600 \mathrm{mg} / \mathrm{m}^{2}$ intravenously every 3 weeks and HER $2^{+}$patients were allowed to continue anti-HER2 therapy with trastuzumab and/or pertuzumab. MRI brain and CT chest/abdomen/pelvis was performed after every two cycles. The study found a $70 \%$ clinical benefit rate (defined as best intracranial partial response $[\mathrm{PR}]+$ stable disease). In 23 evaluable patients with LM, $5(22 \%)$ had a PR and 12 (52\%) had stable disease. The estimated median OS in these patients with LM was 34.6 weeks (95\% CI: 23.3-NE). Kaplan-Meier estimates of median OS in LM breast cancer patients was 8 months compared with historical data of 3.1-3.3 months [3,4]. Of note, not only did ANG1005 show antitumor activity intracranially, there was also disease control in $79 \%$ extracranially. These data are promising since the baseline characteristics of the patients in this study suggest that they were heavily pretreated: $84 \%$ had prior taxane therapy, $84 \%$ had prior intracranial radiotherapy and a median of 1 year since initial diagnosis of BM. The safety and tolerability was consistent with standard taxane therapy with $63 \%$ grade $\geq 3$ neutropenia and lymphopenia, $7 \%$ grade $\geq 3$ peripheral neuropathy and $11 \%$ grade $\geq 3$ fatigue [5]. Even though, data regarding cognitive impairment postANG1005 were not presented, the impressive improvement in OS in LM patients supports the planned Phase III investigation of ANG1005.

In metastatic NSCLC with mutated EGFR (EGFRm), the incidence of BM and LM is $44 \%$ [6]. Data were presented from the Phase I study of AZD3759, the first EGFR inhibitor designed to cross the blood-brain barrier for EGFRm NSCLC (NCT02228369). All 29 patients on the study had progressed after at least one line of EGFR tyrosine kinase inhibitor and one line of chemotherapy and 17 had prior brain radiation therapy. 21 of the 29 patients on the study had measurable BM, and five had LM. Safety and tolerability were the primary objectives and secondary objectives included pharmacokinetic and antitumor efficacy. AZD3759 was well tolerated up to $300 \mathrm{mg}$ twice a day with the most common adverse events being skin rash $(45 \% ; 17 \% \geq$ grade 3$)$ and diarrhea (59\% with $3 \% \geq$ grade 3 ) as previously seen with other tyrosine kinase inhibitors. Sufficient CNS exposure was demonstrated with CSF penetration of AZD3759 at a ratio of 1:1 to plasma and 0.5:1 for its metabolite. Antitumor activity was also seen. Eight of 20 patients with measurable BM had tumor shrinkage by Response Evaluation Criteria in Solid Tumors (RECIST) assessment, and three had confirmed PR. In the five LM patients, all but one patient had at least 50\% decrease in CSF tumor cell number [7]. Updated results from the Phase I study (BLOOM) of AZD3759 or osimertinib at $160 \mathrm{mg}$ once daily also demonstrated safety and tolerability. Additionally, in 20 pretreated patients with EGFRm NSCLC and LM, five had neurological function improvement from baseline, seven had radiological improvements in LM and two patients had clearance of tumor cells from CSF at two consecutive visits. Of the 
15 remaining patients on treatment, seven have been on treatment for over 9 months suggesting durable clinical benefit [8].

While promising new agents like AZD3759 are being further investigated for treatment of LM, the optimal management remains to be understood. Further highlighting that need was the data presented from a systematic review of EMBASE, Ovid MEDLINE ${ }^{\circledR}$, PubMed and the Cochrane Central Register of Controlled Trials. The data from the treatment strategies for LM in breast cancer patients from 1946 to September 2015 were presented. Outcome measures collected were OS, time to neurologic progression, quality of life and treatment toxicity. 181 of 724 studies met the prespecified eligibility criteria. Of the four randomized controlled trials identified, only one specifically compared systemic therapy and involved field radiotherapy to with or without intrathecal methotrexate. There was no benefit to intrathecal chemotherapy. In addition, this study highlighted the lack of wellconducted randomized trials [9]. Prospective trials focusing on tumor type or mutation with good methodology are needed to better understand the risks and benefits associated with the management of patients with LM.

Another common malignancy that spreads to the brain is metastatic melanoma. In the first randomized comparison of first-line immune checkpoint inhibitors, pembrolizumab (an anti-PD-1 therapy) compared favorably to ipilimumab that blocks CTLA-4 (RR: 33 vs 12\%; p < 0.001) [10]. In this study, only $10 \%$ of patients had BM. In the retrospective analysis of 113 consecutive patients treated at Massachusetts General Hospital from 1 May 2014 to 31 October 2015, clinical activity of pembrolizumab was demonstrated in metastatic melanoma patients with
BM. Of the 36 (40\%) patients who had BM prior to starting pembrolizumab, $72 \%$ had prior treatment of their BM. 55.1\% had disease progression on pembrolizumab but the intracranial-only disease progression was only observed in $10 \%$ of the patients. With a median follow-up of 8.5 months, 49 patient $(55.1 \%)$ had disease progression on pembrolizumab and median overall time to progression at any site was 4 months (90\% CI: 2.7-7.8 months). The median time to progression at any site for those with treated BM was the longest at 6.8 months, compared with 5.4 months in patients without $\mathrm{BM}$ and 1.2 months in patients with untreated BM. The 6-month OS estimate was 86\% (90\% CI: 77-91\%) [11]. This retrospective analysis suggests clinical activity of pembrolizumab in metastatic melanoma patients with BM. The early results presented at the last year's American Society of Clinical Oncology annual meeting from the ongoing trial of pembrolizumab in patients with previously untreated or progressing BM showed promising activity in this study population [12]. Accrual continues in this ongoing clinical trial (NCT02085070) and data from this study will no doubt be instrumental in the future management of metastatic melanoma patients.

\section{Financial \& competing interests disclosure}

The authors have no relevant affliations or financial involvement with any organization or entity with a financial interest in or financial conflict with the subject matter or materials discussed in the manuscript. This includes employment, consultancies, honoraria, stock ownership or options, expert testimony, grants or patents received or pending, or royalties.

No writing assistance was utilized in the production of this manuscript.

\section{References}

1 Kayama T, Sato S, Sakurada K et al. JCOG0504: a Phase III randomized trial of surgery with whole brain radiation therapy versus surgery with salvage stereotactic radiosurgery in patients with 1 to 4 brain metastases. J. Clin. Oncol. 34 (Suppl.), Abstract 2003 (2016).

2 Prabhu RS, Press RH, Patel KR et al. Single fraction stereotactic radiosurgery (SRS) alone versus surgical resection and SRS boost for large brain metastases: a multi-institutional analysis. J. Clin. Oncol. 34(Suppl.), Abstract 2076 (2016).
Abouharb S, Ensor J, Loghin ME et al. Leptomeningeal disease and breast cancer: the importance of tumor subtype. Breast Cancer Res. Treat. 146(3), 477-486 (2014).

4 De Avevedo C, Cruz MR, Chinen LT et al. Meningeal carcinomatosis in breast cancer: prognostic factors and outcome. J. Neurooncol. 104, 565-572 (2011).

5 Kumthekar P, Tang SC, Brenner AJ et al. ANG1005, a novel brain-penetrant taxane derivative, for the treatment of recurrent brain metastases and leptomeningeal carcinomatosis from breast cancer. J. Clin. Oncol. 34(Suppl.), Abstract 2004 (2016).
6 Eichler AF, Kahler KT, Wang DL et al. EGFR mutation status and survival after diagnosis of brain metastasis in nonsmall cell lung cancer. Neuro Oncol. 12(11), 1193-1199 (2010).

7 Ahn MJ, Kim DW, Kim TM et al. Phase I study of AZD3759, a CNS penetrable EGFR inhibitor, for the treatment of non-small-cell lung cancer (NSCLC) with brain metastasis (BM) and leptomeningeal metastasis (LM). J. Clin. Oncol. 34(Suppl.), Abstract 9003 (2016).

8 Yang JC, Kim DW, Kim SW et al. Osimertinib activity in patients (pts) with leptomeningeal (LM) disease from non-small 
cell lung cancer (NSCLC): updated results from BLOOM, a Phase I study. J. Clin. Oncol. 34(Suppl.), Abstract 9002 (2016).

9 Optimal management of leptomeningeal carcinomatosis in breast cancer patients - a systematic review. J. Clin. Oncol. 34 (Suppl.), Abstract e13526 (2016).

10 Robert C, Schachter J, Long G et al. Pembrolizumab versus ipilimumab in advanced melanoma. N. Engl. J. Med. 372, 2521-2532 (2015).

11 Dagogo-Jack I, Hurder-Giobbie A, Gainor JF et al. Retrospective analysis of activity of pembrolizumab (pembro) in melanoma patients (pts) with brain metastasis (BM).
J. Clin. Oncol. 34(Suppl.), Abstract 2071 (2016).

12 Kluger HM, Goldberg SB, Sznol M et al. Safety and activity of pembrolizumab in melanoma patients with untreated brain metastases. J. Clin. Oncol. 33(Suppl.), Abstract 9009 (2015). 